\title{
دور المناهج الدراسية نحو بناء مجتمع المعرفة \\ The role of curricula towards building a knowledge society
}

\author{
أ.د/ محمد حماد هندي \\ أستاذ المناهج وطرق التدريس \\ عميد كلية التربية - جامعة بني سويف \\ modhendy@bsu.edu.eg
}


هدفت هذه الورقة البحثية إلى التعرف على ما يمكن أن تقدمه المناهج الدراســـية نحو المســــاهمة في بناء

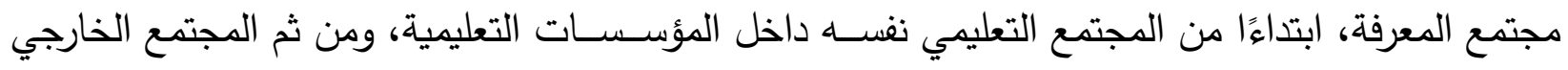
الذي تتواجد فيه تلك المؤسـسـات. وقد جاء محتوى الورقة البحثية تحت محورين أسـاسـيين؛ وهما: مجتمع

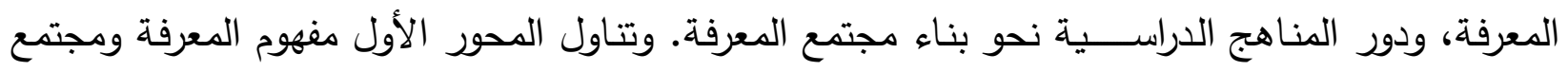

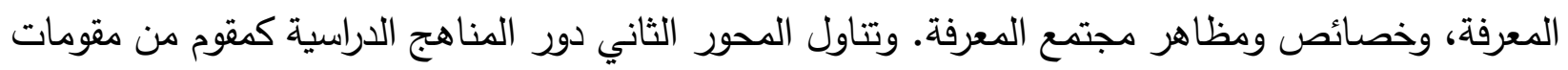

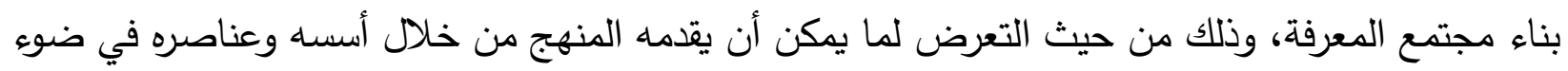

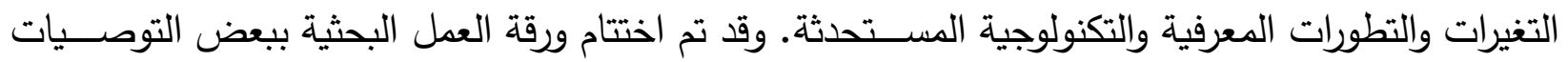
ذات العلاقة بالمحاور المذكورة.

الكلمات المفتاحية: مجتمع المعرفة، المنهج الدراسي وبناء مجتمع المعرفة

\section{Abstract}

This research paper aimed to explain what can curricula present towards the building of the knowledge-based society; either inside or outside of educational institutes. The paper includes two main dimensions which are knowledge-based society and the role of curricula towards the building of knowledge-based society. The first dimension included what is meant by knowledge, knowledge-based society, and characteristics of a knowledge-based society. The second dimension included what can foundations and elements of the curriculum present to help build the knowledge-based society in the light of new knowledge and technological changes and developments. The paper was concluded by presenting some recommendations related to the mentioned dimensions included.

Keywords: Knowledge-based Society, Curricula and building Knowledge-based Society. 


\section{دور المناهج الدراسية نحو بناء مجتمع المعرفة}

رغم شيوع المعرفة وانتشارها عبر الأزمنة والقرون المختلفة، وإدراك أهميتها، وتوظيفها في الحياة؛ وفقًا لما أنتجه المفكرين في ضوء ما فرضته الظروف ووفرته الإمكانات المتاحة عبر تلك الأزمنة والقرون، إلا أننا مازلنا لا نعرف الكثير منها وعنها. وحتى عهد قريب كان مقدار الإلمام بالمعرفة ضئيلا حتى جاءت تكنولوجيا

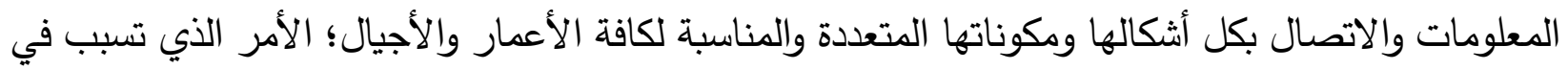

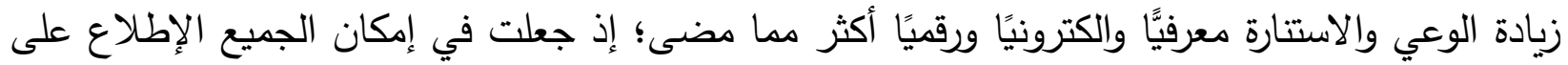
كثير من المعلومات والمعارف، واكتساب ما يرتبط بها من مهارات وجوانب تطبيقة أخرى، الأمر الذي قد تسبب بدوره في تغيير التكوين المعرفي والسلوكي والانفعالي للفرد. والجدير بالذكر أننا على - المستوى القومي - في أثد الحاجة إلى المعرفة واكتسابها وتوظيفها واستثمارها الآن؛ لأنها تمثل سلاحًا هامًا ضد الكثير مما يتم من ممارسات غير لائقة بالعادات والفيم الاصيلة للمجتمع.

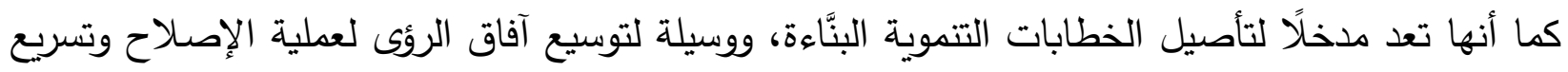
حركة التتمية القومية الثاملة والمستدامة، وأساسًا لفض الاختلافات بين الأفراد، ومن ثم بين المجتمعات (نبيل، 2009)؛ إذ كيف يتم التفاوض، والإقناع، وحل المشكلات المشتركة بدون بناء معرفي شديد ومرن حول تلك

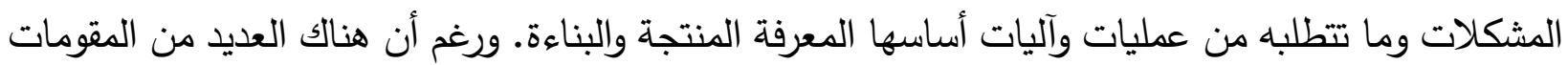

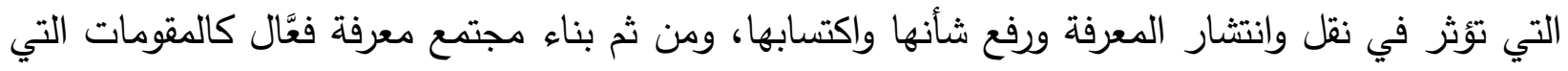
تتعلق باللغة، وجودة المناهج والتعليم، وتوافر المصادر المعرفية، والعوامل الثقافية والاجتماعية، إلا أن المناهج

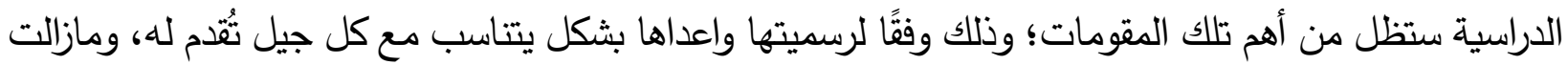
هي الركيزة الأساسية التي تلتف حولها كل الامكانات والمقومات الأخرى في سبيل إعداد الأجيال الحالية

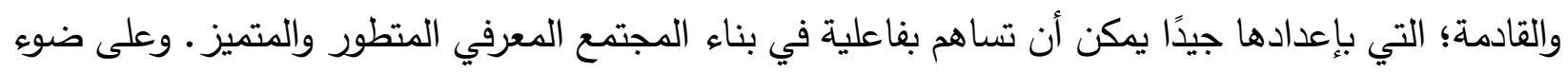
ذلك سوف تتناول هذه الورقة البحثية محورين أساسيين - وما يتبعهما من محاور فرعية- حول مجتمع المعرفة، باءة ودور المناهج الدراسية نحو بناءه وتثكيله. 


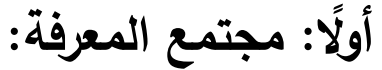

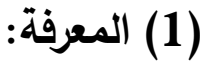

لقد اصبح من شبه المتفق عليه حاليا أن للمعرفة دورًا لا يقل عن رأس المال المادي، وأصبحت بمثابة العامل

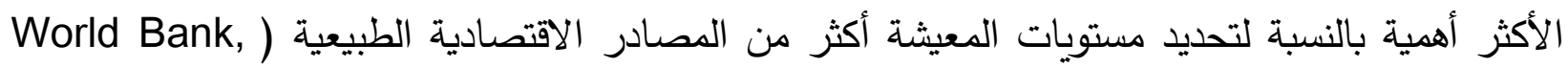

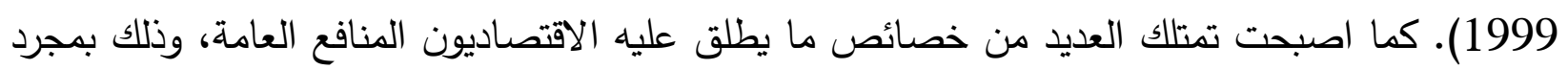
اكتثاف المعرفة ونشرها ومشاركتها بين الأفراد والمجتمعات. وفي الواقع تزداد القيم الاجتماعية والاقتصادية للمعرفة عندما يتم نشرها وتقاسمها مع الآخرين (Stiglitz, 1999).

ومن حيث مفهومها فهي تُعد مفهومًا في غاية التعقيد؛ شأنه في ذلك شأن المفاهيم الأساسية الأخرى حول عالم المعرفة مثل: الوعي، والإدراك، والفهم، والحس، والتفكير ، والذكاء ... إلخ. وربما يرجع السبب الرئيسي وراء

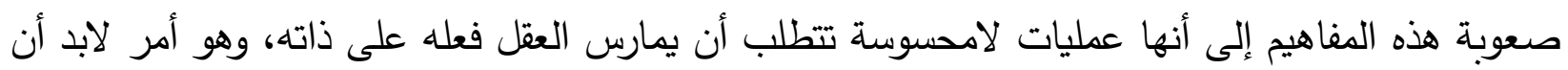
يوقعه في فخ الحلقية؛ ليجد نفسه وقد انتهى به الامر من حيث بدأ (نبيل علي، 2009). وفي هذا الصدد تم

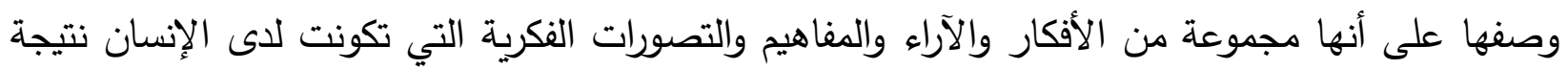

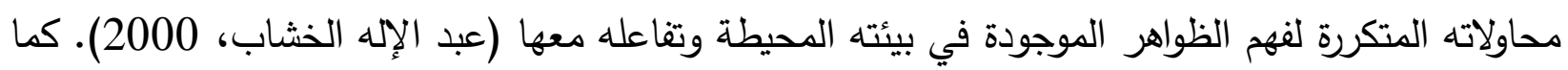

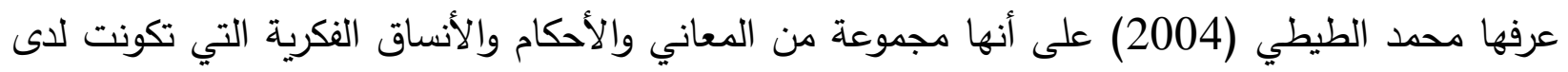

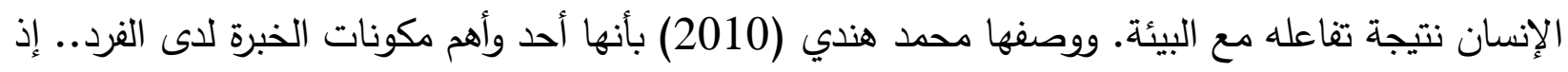

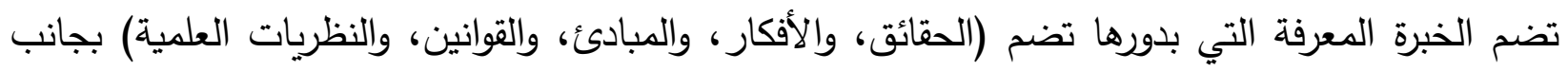

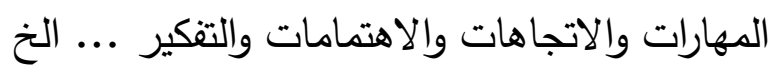

وفيما يتعلق بأهمية المعرفة في الوقت الحالي، فإنها بالفعل قد أصبحت موردًا مهما قد يفوق بأهميته الموارد

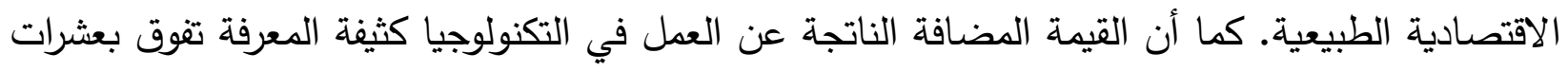
وربما مئات المرات القيمة المضافة الناتجة عن العمل في الصناعات التقليدية.

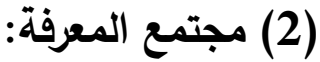

ظهر مصطلح مجتمع المعرفة نتيجة التحول من مجتمع المعلومات إلى مجتمع المعرفة مع نهاية القرن العشرين وبداية القرن الواحد والعشرون نتيجة تغيرات جذرية في جوانب عديدة من أنشطة المجتمع بمجالات

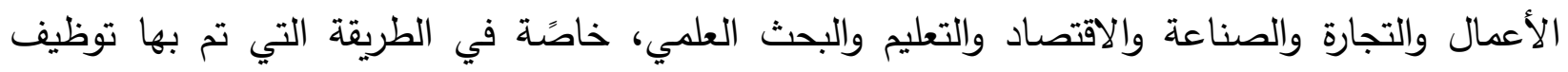
المعلومات وطرق حل المشكلات وسبل البحث لأسئلة عديدة قائمة أو مستحدثة. وفي هذا الصدد تم التمييز

\section{International Journal of Curriculum \& Technological Education}


بين مجتمع المعلومات ومجتمع المعرفة، إذ بثير مجتمع المعلومات Information-based Society إلى الى الماتي المجتمع القائم على أساس جمع المعلومات وفحص مصادرها لاستقاء المزيد منها وتداولها بالتدوين والنسخ والتلقين كوسيلة للتعليم والبحث، بينما يشير مجتمع المعرفة Knowledge-based Society إلى المجتمع

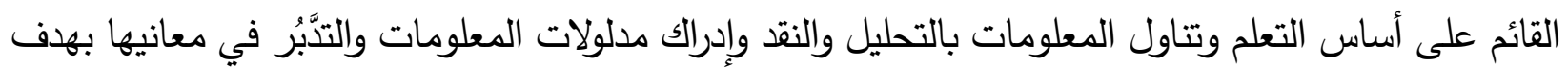
الابتكار والاستنباط (مجلس البحث العلمي، 2004). ويشير مصطلح مجتمع المعرفة أيضًا إلى المجتمع الذي وني تكون المعرفة فيه بمثابة المصدر المبدئي للانتاج افضل من رأس المال المادي. كما يمكن أن يشير إلى ولى المجتمع الذي فيه يصبح نشر المعرفة وانتاجها وتطبيقها هو المبدأ التنظيمي في جميع جوانب النشاط البشري( اليونسكو 2009).

وتم وصف مجتمع المعرفة أيضًا في دراسة زياد بركات وأحمد عوض (2011) بأنه المجتمع الذي يتصف أفراده بامتلاك حر للمعلومات وسهولة تداولها وبثها عبر التقنيات المعلوماتية والحاسوبية والفضائية المختلفة،

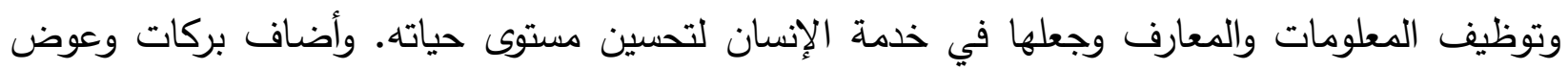

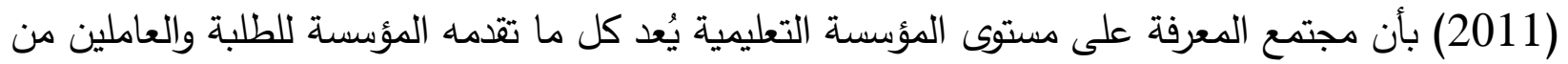
خدمات تزيد من قدراتهم على استخدام التقنيات المعلوماتية المعاصرة بأشكالها المختلفة لزيادة كفاءتهم البحثية والأكاديمية والإدارية. وعلى مستوى الأفراد، يمكن وصف مجتمع المعرفة بأنه مجموعة من الافراد ذوي

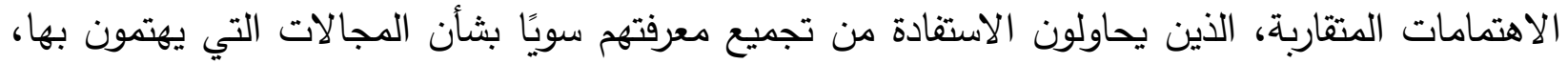
وخلال هذه العملية يضيفون المزيد إلى هذه المعرفة، وهكذا فإن المعرفة هي الناتج العقلي والمُجدي لعمليات الإدراك والتعلم والتثكير • وأشارت منظمة اليونسكو إلى أنه في مجتمع المعرفة تتوفر مصادر الحصول على في أكبر قدر من المعلومات، ويتوفر شكل أفضل للتفاعل الاجتماعي والتعبير الثقافي، وبالتالي يكون لدى الإنى الافراد مزيد من الفرص للمشاركة والتبادل المعرفي (UNESCO, 2004).

(3) (3) خصائص مجتمع المعرفة:

تتميز المعرفة في حد ذاتها بالعديد من الخصائص والسمات؛ لما لها من مكونات عديدة كالحقائق والمعلومات والأفكار والمبادئ والقوانين والنظريات، ومتى أصبحت المعرفة أساسًا لتشكيل مجتمع من المجتمعات؛ فلابد أن تُضفي عليه الكثير من الخصائص والسمات والتي من أهمها: - انشار المعرفة العلمية والمنتجة كنتيجة لانتشار مستحدثات تكنولوجيا المعلومات والاتصال. 
- رقي المؤسسات التعليمية من حيث جودة المقررات الدراسية والبحوث العلمية وارتباطها بسوق العمل، ومن ثم تحولها إلى مؤسسات منتجة للأفكار والخبرات.

- توافر قواعد بيانات معرفية عامة ومتخصصة يُستفاد منها في كافة المؤسسات المجتمعية. - ترابط وتلاحم قوي بين المؤسسات التعليمة والمجتمعية بناءًا على توظيف فعَّال لمصادر المعرفة وما توفره

$$
\text { من معلومات وبيانات. }
$$

- وجود ادرالك معرفي والكتروني ورقمي واسع لدى كل أفراد المجتمع.

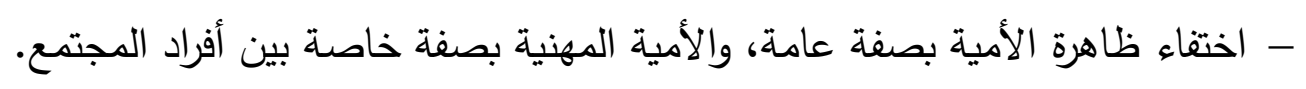
- توافر تعليم تقني عالي وراقي محوره المعرفة المتقدمة والمنتجة.

UNESCO, ) وفي هذا السياق حدد إيفرز (Evers, 2000) خصائص مجتمع المعرفة على النحو التالي :(2004 - تركيز الصناعات فيه على منتجات تعتمد على التكنولوجيا الرقمية والذكاء الاصطناعي. - تحول مؤسساته - الخاصة والحكومية والمجتمع المدني - إلى منظمات تعليمية ذكية. - التميز بزيادة في المعرفة المنظمة في شكل خبرة رقمية مخزنة في بنوك للبيانات. - كثرة مراكز الخبرة وإنتاج المعرفة. - التميز بثقافة معرفية فائقة حول إنتاج المعرفة واستخدامها.

وفي هذا الصدد جاء في دراسة زياد بركات وأحمد عوض (2011) أن مجتمع المعرفة يتميز بقوته

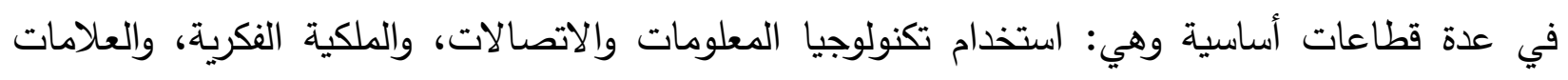
التجارية، والخدمات المالية، وقواعد البيانات، وخدمات الترفيه، والتكنولوجيا الحيوية (مهدي ذياب، ونادية جمال

\section{ثانيًا- دور المناهج الدراسية نحو بناء مجتمع المعرفة:}

رغم تعدد وتتوع المصادر التربوية - كغيرها من المصادر الأخرى - التي يمكن أن تساهم في بناء

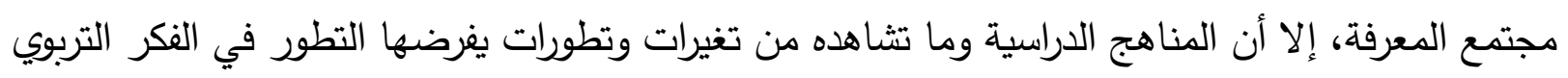

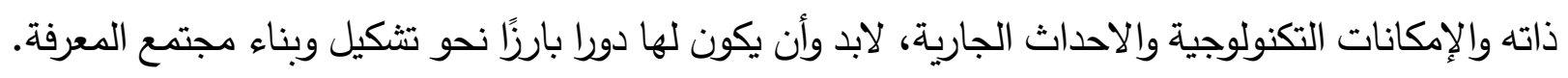

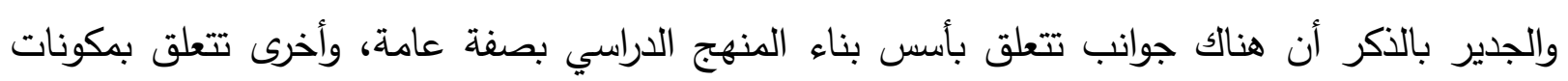
وعناصر المنهج. 


\section{(1) على مستوى أسس بناء المنهج:}

بجانب مراعاة الأسس المعروفة لبناء المنهج الدراسي كالبيئة، والمتعلم، والخبرة ... إلخ، لابد من مراعاة الجوانب الجديدة التالية في ضوء متطلبات بناء مجتمع المعرفة: - مجتمع المعرفة ذاته من حيث مفهومه، وخصائصه، واهم معوقات بناءه، وآليات بناءه.

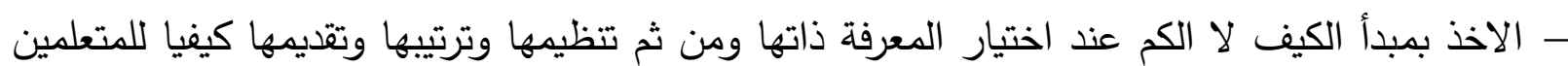
في المراحل الدراسية المختلفة.

- ـكنولوجيا المعلومات والاتصال بصفة عامة كأحد وأهم مقومات بناء مجتمع المعرفة. - المتعلم والخريج في ظل مجتمع المعرفة، بحيث يتمتعا بالقدرة على التفكير المنظم الراقي؛ الذي ترتبط

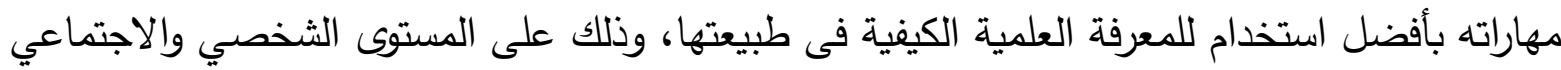
.(Hurd, 1989)

- الاتجاه المستقبلي للمؤسسات التعليمية أو ما يسمى بمدارس المستقبل؛ والتي من ضمن سياساتها التعليمية التحول من التعلم المتمركز حول المادة الدراسية إلى التعلم المتمركز حول المتعلم (راثد الكريم، 2002)، والتركيز على تتمية أنواع جليدة من المهارات بجانب المهارات الأكاديمية (الراسية)؛ مثل: المهارات الثخصية والاجتماعية؛ كمهارات ضبط النفس والسلوك، مع تحمل المسئولية، والتفاعل الناقد مع الآخرين، والعمل من خلال فريق، واحترام قيمة

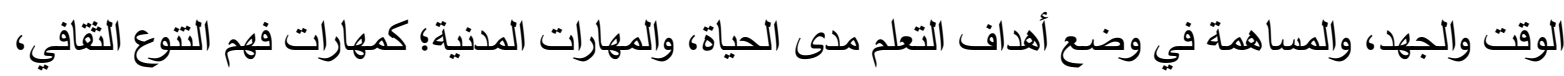
والقدرة على التفاوض وحل النزاعات وفهم الآخرين واحترامهم (مجدي المهدي، 2003). - الاستمرارية .. فيما يتعلق باستمرارية النظر والعمل الدائم حول المنهج بينما يتم تعلمه داخل قاعات التعلم، واستمرارية التطوير على أن يتم التركيز على ما يسمى بالمنهج المُعاش أو المُمارس

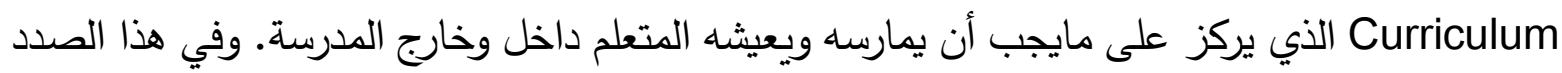

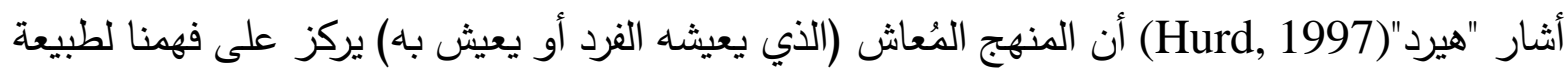
الوجود الإنساني كثديي بيولوجي واجتماعي. والهدف منه هو ربط العلوم والتكنولوجيا مع النمو الثخصي

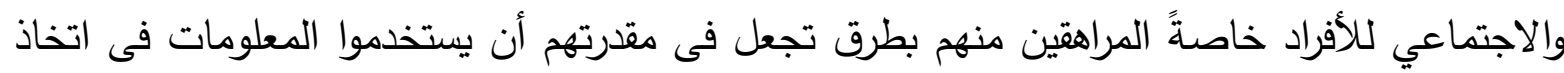
القرار في ضوء ديمقراطية موجهة بالعلم والتكنولوجيا. 


\section{(2) على مستوى عناصر المنهج:}

\section{(أ) بالنسبة لأهداف المنهج:}

لم يَعْدْ مجرد تعليم المعلومات الأساسية والتدريب على استخدامها بهدف مجرد التعايش هو الهدف

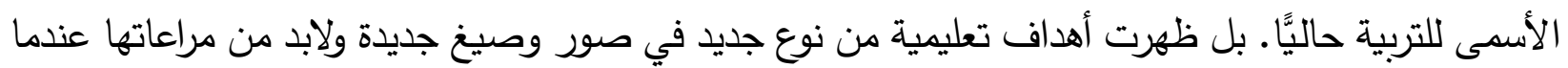
يراد مساهمة المنهج في بناء مجتمع المعرفة. ولعل من أهم أولويات تلك الأهداف هو صياغة فيلة أهداف التعلم ذاتها في صورة مكونات للسلوك تتطلب من المتعلم المرور بخبرات مربية سليمة أول مكوناتها المعرفة بما هاه

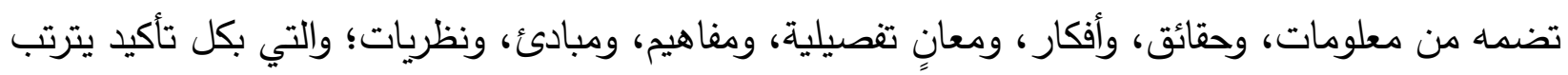

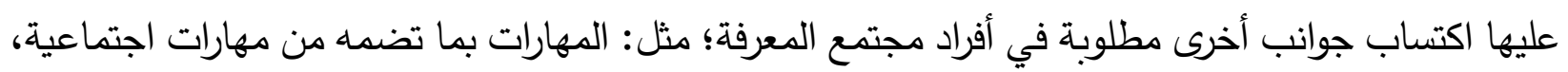
وعقلية، وحركية، والعادات بما تضمه من عادات اجتماعية، وعقلية إيجابية، والقيم والمثل العليا بما تضمه من

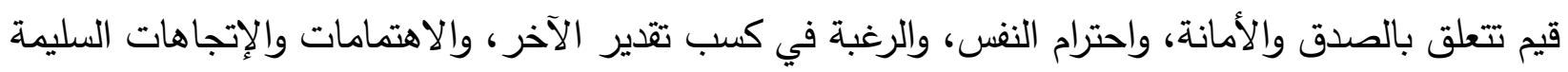

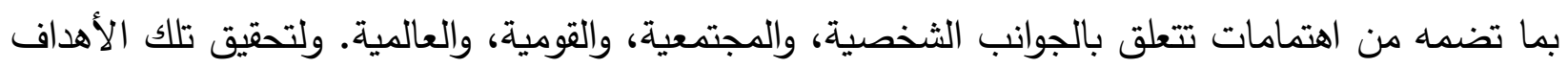
لابد من صياغتها بثكل اجرائي وواقعي عند وضع المنهج المطلوب للمساهمة في تثكيل مجتمع المعرفة.

(ب) بالنسبة لمحتوى المنهج: (ب)

في سياق اهتمام هذه الورقة البحثية لابد من تركيز محتوى المنهج على مبدأ الكيف عند اختيار المعرفة

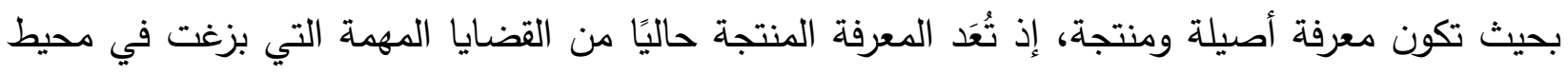

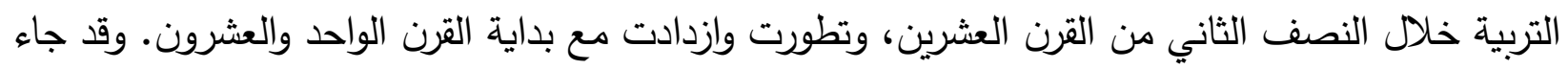
الاهتمام بها نتيجة الثعور بعدم جدوى التحصيل الدراسي السطحي في ضوه استخدام المحتوى الكمي التقليدي

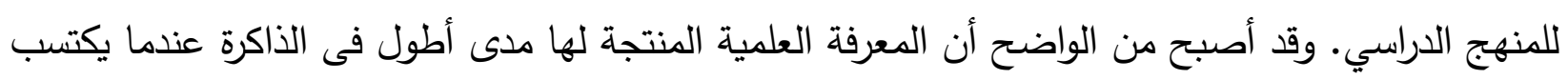
المتعلمون خبرة حولها، ويدركون أن المعرفة يمكن أن تُستخدم فى مساعدتهم على فهم أنفسهم، كما تُستخدم

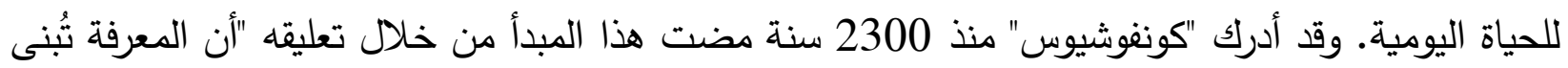

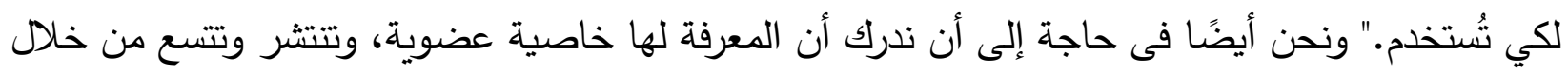

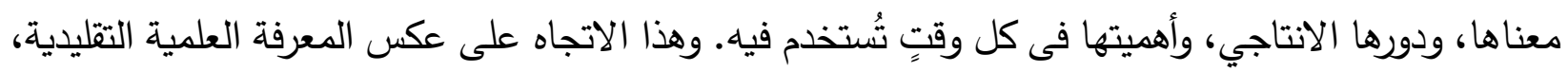
التي تُعَد معزولة فى تخصصات منفصلة مع تطبيق أقل خارج قاعة الدراسة، ومن ثم النظر إليها وكأنها في معطفها الضيق في حين أن هنالك حاجة ماسة الآن لأخذ الخبرات الحياتية فى الاعتبار (Hurd, 1997). 
وفي هذا السياق أكدت "لين أولسون" (2000) أنه يجب أن نزيد من التركيز على خبرات التعلم الحياتية لدى المتعلمين داخل المدرسة؛ حتى يرتفع مستوى جودتها وجودة الخريجين المطلوبين لعالم اليوم. (ج) بالنسبة لطرق التعليم والتعلم: (ج)

ترتب على التغيرات والتطورات السريعة التي حدثت ومازالت تحدث في المجالات الثقافية والعلمية والتكنولوجية تغيُرات في سلوك الأفراد، وكيفية تعاملهم مع بعضهم البعض من ناحية، ومع الحياة بجوانبها

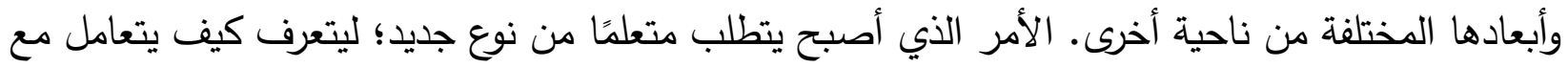

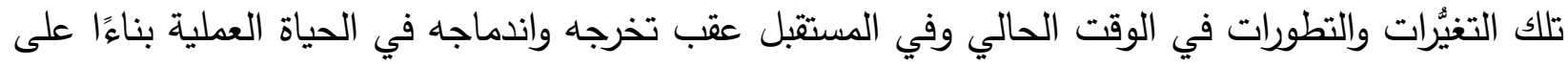
المستحدثات المعرفية والتكنولوجية .. متعلمًا لله القدرة على التفكير المنظم والناقد، وقراءة وتحليل المواقف التي يتواجد فيها والتعامل معها، خاصةً وأن تلك المواقف يغلب عليها الطابع العلمي والتكنولوجي (محمد هندي، 2010). وعلى ضوء ذلك لابد أن يساعد المنهج الدراسي في ذلك من خلال مراعاة تضمن واستخدام مداخل وطرق واستراتيجيات تعليم وتعلم تركز على التفكير، وحل المشكلات، وتتمية أنماط التعلم، ومهارات وعادات

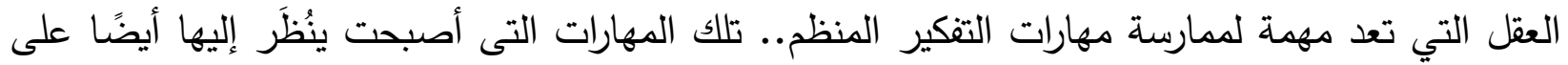

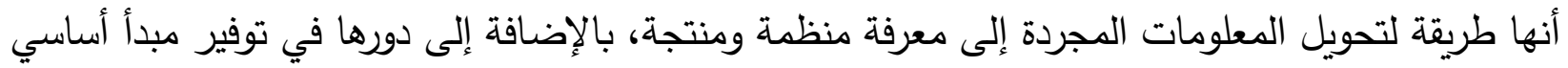

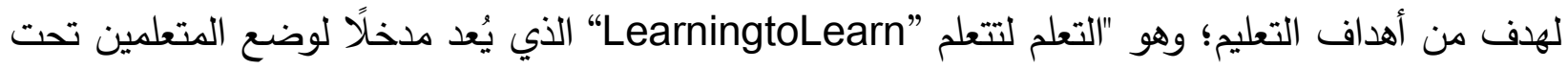
رهن استعداداتهم العقلية خلال فترات حياتهم. (د) بالنسبة لوسائل ومصادر التعلم: بجانب استخدام كل وسائل ومصادر التعلم البسيطة والواقعية والملموسة، لابد من الاهتمام ببصادر وتكنولوجيا التعليم المستحدثة لتتناسق مع ما يوضع من أهداف متتوعة ومتطورة للمنهج من ناحية، وللعمل على اكتمال اكتساب الخبرة في شكلها الجديد الذي يتفق ومتطلبات عالم ومجتمع المعرفة الجديد من ناحية أخرى. فمن المؤكد أن تحقيق مثل تلك الأهداف المستحدثة للمنهج تحتاج إلى بيئة تعليمية غير تتليدية .. بيئة تعليمية ثُنبى كل طرقها واستراتيجياتها على استخدام التطبيقات التكنولوجية المستحدثة التي تثجع على التفاعل التى الصفي؛ مثل: التعلم الإلكتروني بصادره المختلفة (كالتعلم بالكمبيوتر، واستخدام الإنترنت، والتجارب الافتراضية...إلخ). ذلك الأمر الذي يحتم ضرورة السعي نحو بناء مجتمعات معرفية فعَّالة أساسها تكنولوجيا المعلومات والاتصال والتعليم عن بُعد من خلال المستحدثات التكنولوجية (محمد هندي، 2010). 


\section{(ه) بالنسبة لتقويم المنهج:}

على ضوء الحاجة إلى بناء مجتمع المعرفة لابد من دور جديد للتقويم ليكون عملية نشطة مكملة لإجراءات

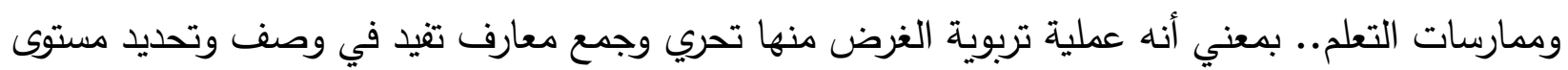
إنجاز المتعلم في ظل مجتمع المعرفة. ويشير "إينجر" وياجر" (Enger\& Yager, 2001) إلى أن كل الإجراءات والممارسات التقييمية بغض النظر عن نوعها ومستوياتها لابد وأن ترتبط بنواتج التعلم المرجو

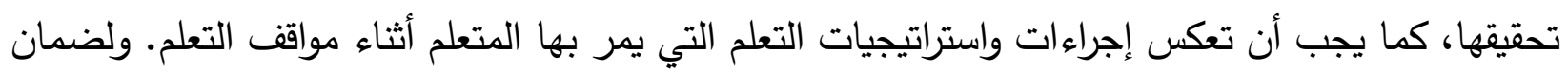
اكتساب المعرفة المنتجة وتطبيقها في مجتمع المعرفة لابد من مراعاة التقييم الأصيل والتقييم المبني على الأداء؛

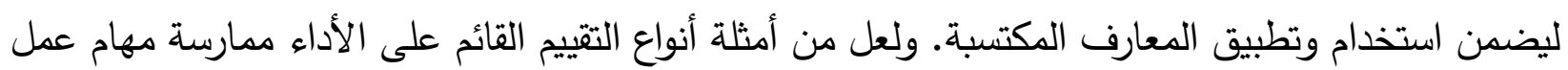
أدائية حقيقية متعددة بغرض الحصول على وتطبيق المعرفة، والمشروعات قصيرة وطويلة المدى الفردية والجماعية، والملاحظة المباشرة، والأسئلة مفتوحة النهاية، وبناء خرائط المفاهيم، والعصف الذئئ الذهني، والتقارير المعرفية الذاتية، والبورتفوليو، وقوائم تقييم دهام الأداء، وقواعد التقدير المتدرج... إلخ. وبذلك يتضح وأنة أن استخدام

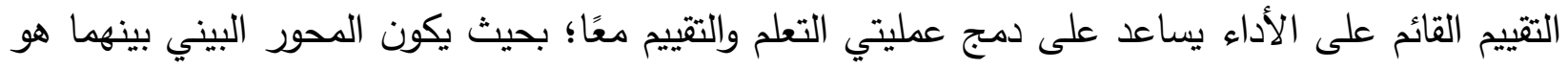

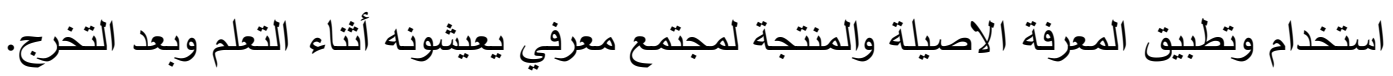

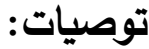

على ضوء ما جاء عاليه تحت محاور هذه الورقة البحثية ، يُوصى بما يلي:

- إعادة النظر أولاً بأول حول المناهج الدراسية بالمؤسسات التعليمية ودراسة مدى تبنيها لأسس جديدة لتخطيطها ووضعها في ضوء متطلبات مجتمع المعرفة. - التركيز على إمداد المؤسسات التعليمية بأحدث وسائل تكنولوجيا المعلومات والاتصال، مع تطوير مناهجها بحيث تتناسب وتلك التكنولوجيا من ناحية، ومتطلبات مجتمع المعرفة من ناحية أخرى.

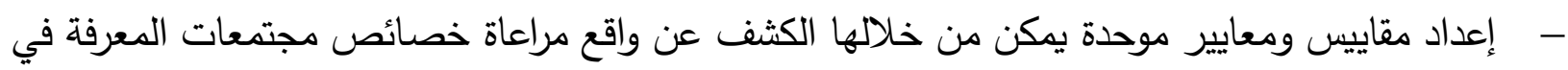
المناهج، ومن ثم اقتراح الاستراتيجيات المناسبة لتضمينها. - - اجراء المزيد من الأبحاث والدراسات وأوراق العمل البحثية حول مجتمعات المعرفة، والتتمية المعرفية،

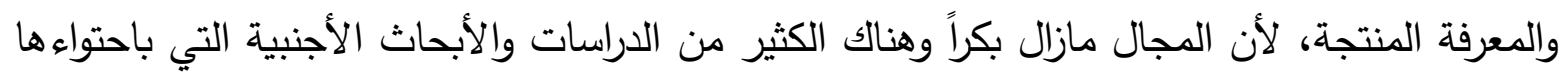
في الدراسات العربية يمكن التثقيف بتلك المجالات لاى كل المستويات العمرية داخل المجتمعات العربية. 


\section{المراجــــــــع العربية والاجنبية}

المراجع العربية:

راشد العبد الكريم (2002). "مدرسة المستقبل". بحوث ندوة مدرسة المستقبل المنعقدة بكلية التربية - جامعة الملك سعود، في الفترة من 22 - 23 اكتوبر ، المجلد الأول، كلية التربية- جامعة الملك لكورئه

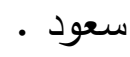

زياد بركات، أحمد عوض (2011).واقع الجامعات العربية في تنمية مجتمع المعرفة من وجهة نظر

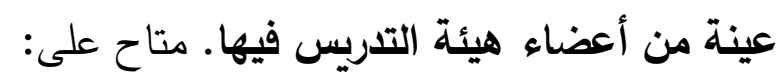

http://www.qou.edu/arabic/researchProgram/researchersPages/ziadBaraka/r1 _drZiadBarakat.pdf

• عبد الإله الخشاب (2000). الجامعة في خدمة مجتمع المعرفة. الاسكندرية: مطبعة جامعة الاسكندرية.

$$
\text { • كرم أبو حلاوة (بدون ). أين العرب من مجتمع المعرفة. متاح على: }
$$

http://www.mokarabat.com/mo10-21.htm

• لين أولسون (2000). ثورة في التعليم: من المدرسة إلى العمل. ترجمة شكري عبد المنعم مجاهد. القاهرة: الجمعية المصرية لنشر الثقافة والمعرفة العالمية.

مجدي صلاح المهدي (2003). "معالم فلسفة تربوية مقترحة لمدرسة المستقبل في ضوه بعضه بعض العاديه

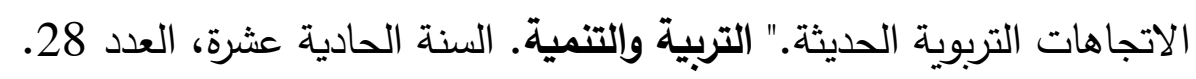
مجلس البحث العلمي بجامعة الملك عبد العزيز (2004). نحو مجتمع المعرفة. سلسلة دراسات يصدرها مجلس البحث العلمي. جدة.

محمد الطيطي (2004). البنية المعرفية لاكتساب المفاهيم تعلمها وتعليمها. عمّان: دار الامل

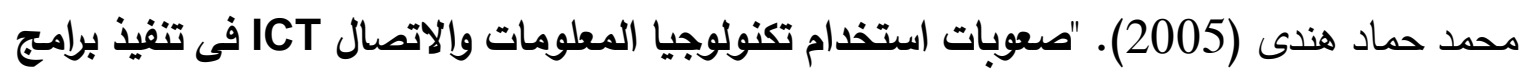

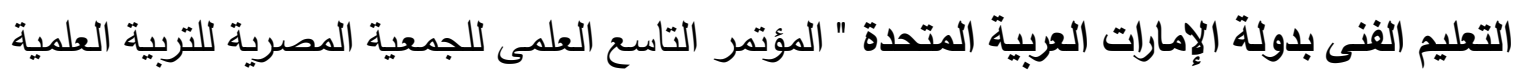

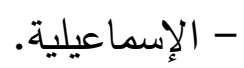
محمد حماد هندي (2010). التعلم النشط: اهتمام تربوي قديم حديث. الطبعة الأولى. القاهرة: دار النهضة العربية. International Journal of Curriculum \& Technological Education 
• مهدي ذياب، ونادية جمال (2006). الجامعة ومجتمع المعرفة - التحدي والاستجابة. الاسكندرية: المكتب الجامعي الحديث.

• نبيل علي (2009). العقل العربي ومجتمع المعرفة : مظاهر الأزمة واقتراحات بالحلول. الجزء الثاني.

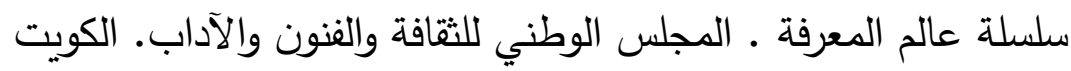

• -محمد حماد هندي (2013). دور دور تكنولوجيا المعلومات والاتصال تجاه تثكيل مجتمع معرفة والته

عربي. ورقة عمل قدمت إلى الم الملتقى الدولي حول " مجتمعات المعرفة" بجامعة جيجل بالجزائر ، نوفمبر 2013.

المراجع الأحنبية:

- Hurd, P. D. (1989b). Science Education and the Nation's Economy. In A, B, Champagne, B. E. Lovitts, \& B.J. Calinger (Eds.) This Year in School Science, Scientific Literacy. Washington. Dc: American Association for the Advancement of Science.

- Hurd, Paqul (1997) Inventing Science Education for New Millennium. Colombia: College Teachers Press.

- Stiglitz, E. J. (1999). Public policy for a Knowledge Economy, Speech, Remarks at the Department Trade and Industry and Center for Economy, Providence, Ri: Brown University Lmic Policy Research. London: The World Bank Group.

- UNESCO (2004). Asia and Pacific Regional for Education. Guidebook 1-ICT in Education and School nets. Available at:

- Enger, S.\& Yager, R. (2001). Assessing Student Understanding in Science: A Standards-Based

- Hendy, M. Hammad (2016). Mid-Egypt Professors and Students Perceptions of the University as A knowledge Society. Paper presented in International Conference on Education and Social Sciences in Singapore (February 18-20, 2016).

- http://www.unescobkk.org/fileadmin/user_upload/ict/ebooks/ScoolNetKit/gui debook1.pdf

- K-12 Handbook. Thousand Oaks, California: Corwin Press, Inc.

- -World Bank (1999). The Power and Reach of Knowledge. World Development Report 1998/1999. P 16-25. 